\title{
Determining local geometrical features of grain boundaries from microscopy
}

\author{
François A. Lavergne, Dirk G. A. L. Aarts and Roel P. A. \\ Dullens \\ Department of Chemistry, Physical and Theoretical Chemistry Laboratory, \\ University of Oxford, South Parks Road, Oxford, OX1 3QZ, United Kingdom \\ E-mail: francois.lavergne@chem.ox.ac.uk
}

\begin{abstract}
Grain boundaries are solid-solid interfaces whose dynamics is driven by their local curvature. As they are fluctuating interfaces and have a width comparable to the lattice spacing of the surrounding grains, the determination of their local geometrical characteristics is difficult. Here we present a method to determine the local normal direction, tangent plane and curvature of grain boundaries from microscopy images using point sampled surface analysis techniques. We apply our algorithm to study the boundary of a shrinking grain in a two-dimensional colloidal polycrystalline material. Our method is easily generalised to three dimensions, which makes it applicable to the wide range of interfaces encountered in soft matter.
\end{abstract}

\section{Introduction}

A grain boundary (GB) is the interface between two adjacent crystallites with differing orientations [1]. Polycrystalline materials consist of many differently oriented crystallites, or grains, in which the network formed by the GBs coarsens to lower the total interfacial area [2]. In this process, known as grain growth, some grains grow at the expense of other ones leading to an increasing average grain size. The origin of grain growth can be traced back to the motion of curved GBs along their normal directions to reduce their local curvature $[2,3]$. The local normal velocity $v$ of a migrating GB is then given by the Herring's relation $[2,4]$

$$
v=M \Gamma \kappa,
$$

where $M$ is the mobility of the grain boundary, $\Gamma$ the GB stiffness and $\kappa$ the local curvature. While $M$ and $\Gamma$ can be directly obtained from the capillary fluctuations of flat GBs $[5,6,7,8]$, directly testing the Herring's relation requires detailed knowledge of the local geometrical features of the GB, and in particular its local curvature.

Experimentally, GBs in atomic systems are typically studied using high-resolution electron microscopy techniques $[9,10,11]$. Though they provide detailed insight into the structural properties of GBs, directly accessing the interface dynamics, including 
curvature driven migration, is generally problematic due to the short time and length scales [9]. Colloidal model systems have been proven very useful in microscopy based single-particle level studies of GBs $[8,12,13,14,15,16,17,18,19]$. However, at this scale, GBs have a width of a few particle diameters and exhibit deformations due to thermal fluctuations $[5,6,7,8,12,20]$. The particles belonging to the GB thus form a noisy discrete cloud of points from which the local geometrical features of the embedded idealised continuous GB are to be extracted.

Detecting and extracting the geometrical characteristics of an interface of unknown shape can be done using various techniques, depending on the dimension and topology of the interface and the spatial resolution of the data. Interfaces can be detected using the variations of the intensity profile on raw images [21], snake algorithms [22], lattice based methods [23], the level set method [24] or particle-based criteria [8, 12, 25, 26, 27]. Specific geometrical features, such as the normal and tangent planes, and the curvature of fluid-fluid interfaces are usually determined using lattice based methods [22, 24] although they are more adapted to simulations where the rules of evolution of the interface are imposed. In experiments, where interfaces are sampled by a discrete cloud of points, the local curvature can be extracted using circular [22] or parabolic fits [28] to the data points, though this only gives reliable results as long as the noise level is low.

The noisy nature of fluctuating GBs therefore makes determining its local geometrical features from microscopy images cumbersome and clearly a robust analysis method is required for this purpose. In this article, we present a relatively simple method that enables us to fully quantify the local geometrical features of GBs sampled by discrete points obtained from microscopy images. We will first outline our method for one-dimensional (1D) interfaces in 2D systems. We test our method on artificially generated and experimental GBs in 2D colloidal crystals. Finally, our method is applicable to other types of interfaces and easily extendable to 3D systems, which broadens its relevance to the wide field of interfaces in soft matter.

\section{Geometrical analysis of point sampled 1D interfaces}

We consider a cloud of points in $2 \mathrm{D}$ space which samples a $1 \mathrm{D}$ interface $L$, whose parametrisation is unknown (see figure 1a). The purpose is to recover the local geometrical features of $L$ from the discrete cloud of points. We first show how to determine the local normal and tangent directions to $L$ using standard Principal Component Analysis (PCA) [29, 30, 31]. Then we will compute the local curvature along the interface using two different methods.

\subsection{Local normal and tangent directions}

For a given point $P$ in the cloud sampling the 1D interface, we define the neighbourhood of $P$ as being the set $\Omega_{P}$ containing the $m$ points located within a distance $r_{c}$ from $P$ (see 
figure 1a). We define the $m$-dimensional vectors $\mathbf{x}=\left(x_{1}, \ldots, x_{m}\right)$ and $\mathbf{y}=\left(y_{1}, \ldots, y_{m}\right)$, where $\left(x_{i}, y_{i}\right)$ are the coordinates of a point $i$ belonging to $\Omega_{P}$. Note that in the following, the notation $\mathbf{x}$ refers to $m$-dimensional vectors while $\vec{u}$ refers to vectors in the $2 \mathrm{D}$ plane. The covariance matrix $C_{P}$ of the coordinates of the points within the neighbourhood of $P$ is defined by $[29,30,31]$

$$
C_{P}=\left[\begin{array}{ll}
\operatorname{Cov}(\mathbf{x}, \mathbf{x}) & \operatorname{Cov}(\mathbf{x}, \mathbf{y}) \\
\operatorname{Cov}(\mathbf{y}, \mathbf{x}) & \operatorname{Cov}(\mathbf{y}, \mathbf{y})
\end{array}\right],
$$

where

$$
\operatorname{Cov}(\mathbf{x}, \mathbf{y})=1 / m \sum_{i=1}^{m}\left(x_{i}-\bar{x}\right)\left(y_{i}-\bar{y}\right)
$$

is the covariance of the two vectors $\mathbf{x}$ and $\mathbf{y}$, with $\bar{x}=1 / m \sum_{i=1}^{m} x_{i}$. Because the covariance matrix is symmetric, it has two positive eigenvalues with two associated orthogonal eigenvectors. The normalised eigenvector associated to the lowest eigenvalue of $C_{P}$ is the normal vector to the interface at $P, \vec{n}_{P}$, while the other normalised eigenvector corresponds to the tangent vector at $P, \vec{u}_{P}[29,30,31]$. By performing this operation for all the points $P$ in the cloud, we obtain all the corresponding normal and tangent vectors along the $1 \mathrm{D}$ interface.

As the normal vector at $P$ can be oriented in two different directions, it is necessary to enforce a consistent orientation along $L$ so that for two neighbouring points $P$ and $Q, \tilde{\mathbf{n}}_{\mathbf{P}} \cdot \tilde{\mathbf{n}}_{\mathbf{Q}} \geq \mathbf{0}[31,32]$. In simple cases, this can be achieved by forcing the normals to point towards a common observation point located at $\vec{v}_{0}$, far away from the interface [32]. Equivalently, this implies that every point $P$ with position $\vec{r}_{P}$ and normal vector $\vec{n}_{P}$ has to satisfy:

$$
\vec{n}_{P} \cdot\left(\vec{v}_{0}-\vec{r}_{P}\right)>0 \text {. }
$$

If a common observation point cannot be defined, more sophisticated methods based on graph theory can be used [31]. Finally, the condition $\operatorname{det}\left(\vec{u}_{P}, \vec{n}_{P}\right)=1$ fixes the direction of the tangent vector $\vec{u}_{P}$ as shown in figure $1 \mathrm{~b}$.

\subsection{Local curvature}

Next we determine the local curvature $\kappa_{P}$ at every point $P$ in the cloud sampling the 1D interface $L$ using two different methods that we will compare in the following. The first one is based on the osculating parabola (OP) of the points in $\Omega_{P}$. First, we change the reference frame to the local tangent frame $\left(\vec{u}_{P}, \vec{n}_{P}\right)$ centred at $P$ : the new coordinates of the points in $\Omega_{P}$ are now denoted $\mathbf{u}=\left(u_{1}, \ldots, u_{m}\right)$ and $\mathbf{n}=\left(n_{1}, \ldots, n_{m}\right)$. In the local frame, the coordinates of $P$ are $(u, n)=(0,0)$ and the interface $L$ is locally described by the equation $n=f(u)$ where $f$ is a function such that $f(0)=f^{\prime}(0)=0$ (see figure 1c) [33]. Here, $f^{\prime}$ is the derivative of $f$ with respect to $u$. As a result, to the lowest order in $u, f$ reads

$$
f(u)=\frac{1}{2} f^{\prime \prime}(0) u^{2}+\mathcal{O}\left(u^{3}\right),
$$


(a)

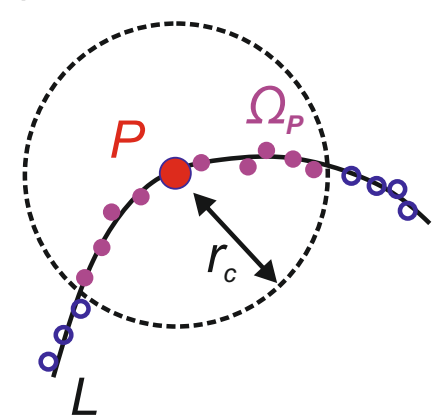

(d)

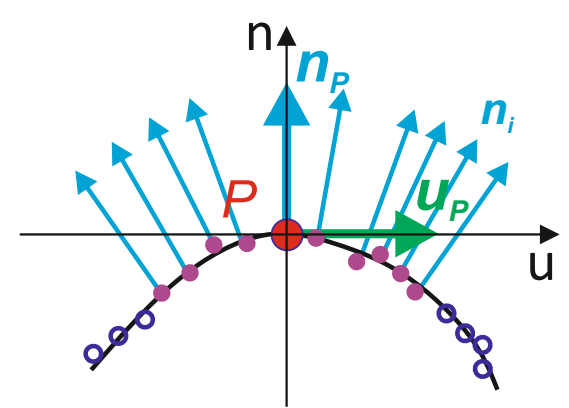

(b)

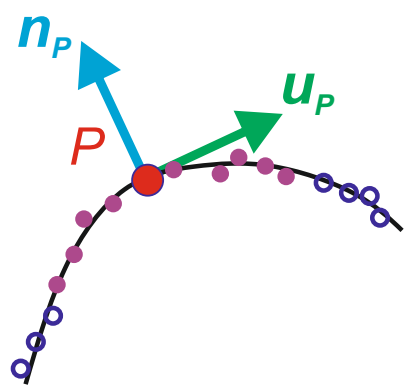

(e)

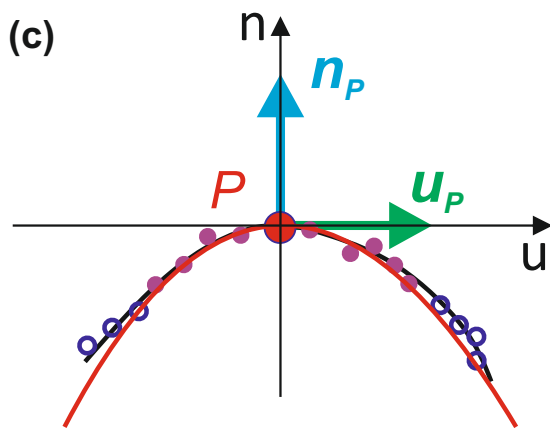

Figure 1. (a) Local view of a $1 \mathrm{D}$ interface $L$ sampled by a cloud of points. The neighbourhood of a point $P$ is the set of points $\Omega_{P}$ within a distance $r_{c}$ from $P$. (b) The tangent and normal unit vectors, $\vec{u}_{P}$ and $\vec{n}_{P}$ at $P$. (c) The osculating parabola $n=1 / 2 a u^{2}$ (red line) in the local tangent frame $\left(\vec{u}_{P}, \vec{n}_{P}\right)$ of $P$. The local curvature is given by $a$ which is negative here. (d) The variation of the normal vectors $\vec{n}_{i}$ within $\Omega_{P}$. (e) The variation of $\phi_{i}=\vec{n}_{i} \cdot \vec{u}_{P}$, as a function of $u_{i}$, the coordinate along $\vec{u}_{P}$. The gradient of the line is $-\kappa_{P}$.

and the osculating parabola to $L$ is given by $n=\frac{1}{2} f^{\prime \prime}(0) u^{2}$. The local curvature at $P$ is thus $\kappa_{P}=f^{\prime \prime}(0)$ [33]. Hence, fitting a function of the form $n=\frac{1}{2} a u^{2}$ to the points $(\mathbf{u}, \mathbf{n})$ yields the local curvature as $\kappa_{P} \simeq a$, see figure 1c.

The second method to determine the local curvature $\kappa_{P}$ is based on the variation of the normal direction along the interface within $\Omega_{P}$. Though similar methods have been used in surface feature detection, they are computationally expensive [34] or give dimensionless curvature indicators [29, 35]. Here we introduce a normal variation (NV) method that is fairly easy to implement and fast to apply to large data sets. In the local tangent frame, we introduce the map $\phi$, which tracks the variation of the normal $\vec{n}(u)$ along the tangent direction at $P, \vec{u}_{P}$ (see figure $1 \mathrm{~d}$ ):

$$
\phi: u \mapsto \phi(u)=\vec{n}(u) \cdot \vec{u}_{P}
$$

If $L$ is locally flat, then $\phi$ is zero as $\vec{n}$ is perpendicular to $\vec{u}_{P}$. If $L$ is positively (negatively) curved, $\phi$ decreases (increases) with $u$ as illustrated in figure 1e. As $\phi$ vanishes at $P$, i.e. $u=0$, its expression to the lowest order in $u$ is given by:

$$
\phi(u)=\phi^{\prime}(0) u+\mathcal{O}\left(u^{2}\right)
$$


and the local curvature is $\kappa_{P}=-\phi^{\prime}(0)$ (see Appendix A). Fitting a function of the form $\phi=\alpha u$ to the points $(\mathbf{u}, \phi)$, where $\phi=\left(\phi_{1}, \ldots, \phi_{m}\right)$ with $\phi_{i}=\vec{n}_{i} \cdot \vec{u}_{P}$, directly leads to the local curvature via $\kappa_{P} \simeq-\alpha$. An example for $\kappa_{P}<0$ is shown in figure 1e.

The method to find the local normal and tangent directions and both methods to determine the local curvature can be straightforwardly extended to surfaces of any dimensions. The details for two-dimensional surfaces in 3D space can be found in Appendix B.

\section{Validating the method on artificial grain boundaries}

\subsection{Generating the artificial grain boundary}

First, we validate the method by applying it to an artificial grain boundary of known curvature. We generate two overlaying hexagonal lattices of orientations $\theta_{1}=15^{\circ}$ and $\theta_{2}=40^{\circ}$. Then we plot a curve $y=-A \sin (2 \pi x / \lambda)$ on top of the two hexagonal lattices and the sites of the lattice of orientation $\theta_{1}\left(\theta_{2}\right)$ located below (above) the curve form the grain $G_{1}\left(G_{2}\right)$. During this process, we removed all the overlapping particles. As a result, we have generated a grain boundary between $G_{1}$ and $G_{2}$, with analytically known shape and, hence, local curvature (see figure 2a). The parameters $A$ and $\lambda$ are set to $A=12 \sigma$ and $\lambda=20 \sigma$, where $\sigma$ is the lattice spacing.

To generate the cloud of points that sample the grain boundary, we first select the lattice sites belonging to the edges of $G_{1}$ and $G_{2}$ enclosing the grain boundary (see the red and blue stars in figure $2 \mathrm{a}$ ). To ensure that the cloud of points samples the grain boundary, which is located in between the edge sites of $G_{1}$ and $G_{2}$, we generate interpolating sites in between consecutive edge sites (see the magenta dots in the inset of figure 2a). Hence, the final cloud of points sampling the grain boundary consists of the edge and the interpolated sites.

As real grain boundaries exhibit thermal noise, the corresponding cloud of points can be quite wide. To assess the effect of noise on the analysis method, we smooth the cloud of points sampling the artificial grain boundary by replacing every point $P$ in the cloud by the centre of mass of all the points situated within a cut-off radius $r_{0}$ from $P[30]$.

\subsection{Geometrical features of the artificial grain boundary}

First, we smooth the cloud of points using a cut-off radius of $r_{0}=2.5 \sigma$ and then we compute the normal and tangent directions of the artificial grain boundary using PCA with a neighbourhood cut-off radius of $r_{c}=2.5 \sigma$ (see section 2.1). We ensure that the normal is consistently oriented along the grain boundary by positioning the common observation point far up in the top half-plane. Next, the local curvature is determined using both the OP and the NV methods (see section 2.2). In figure $2 \mathrm{~b}$ we show the smoothed cloud of points, which are coloured according to the local curvature determined using the NV method, and the normal vectors. The smoothed cloud of 
(a)

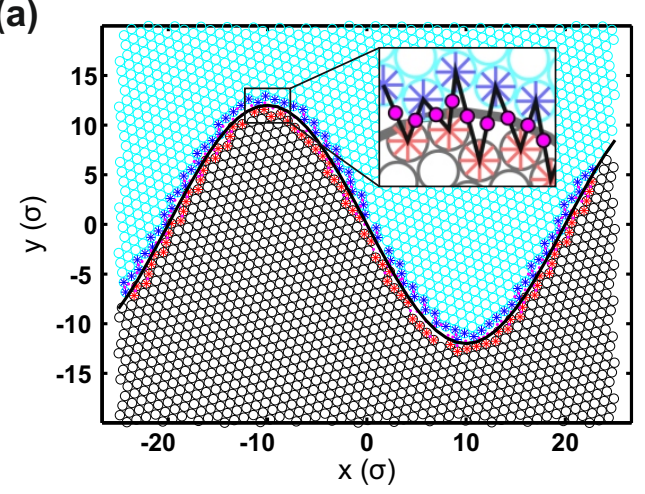

(c)

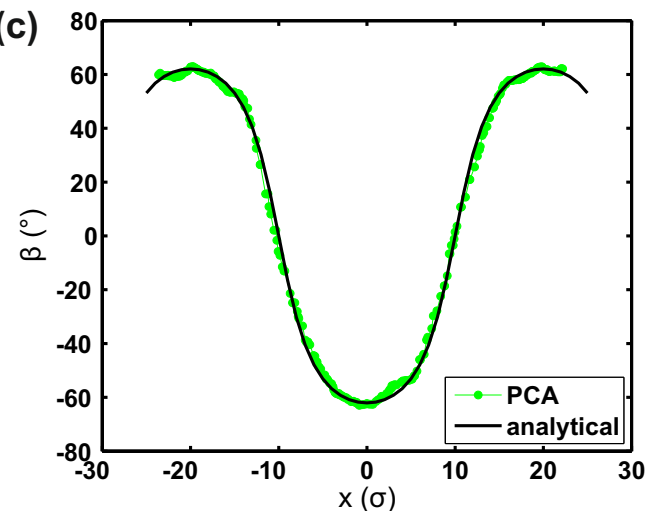

(e)

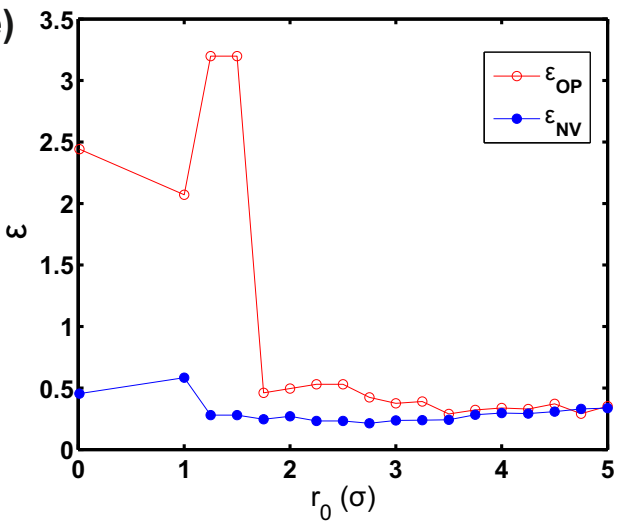

(b)

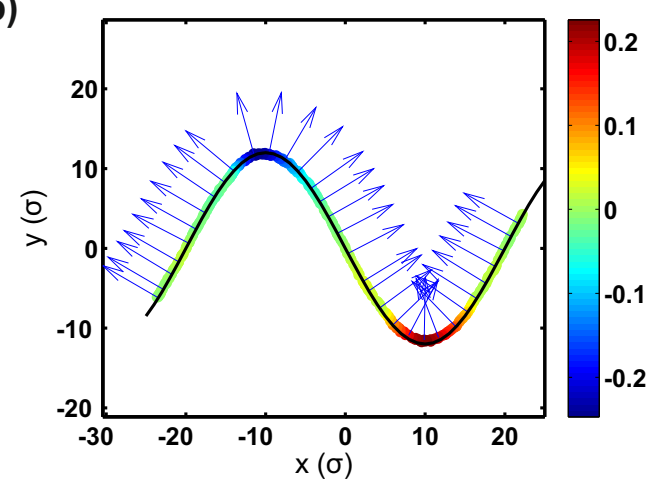

(d)

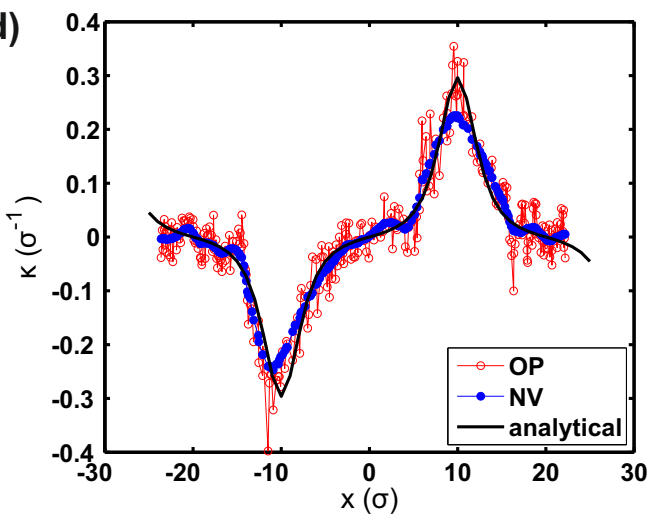

(f)

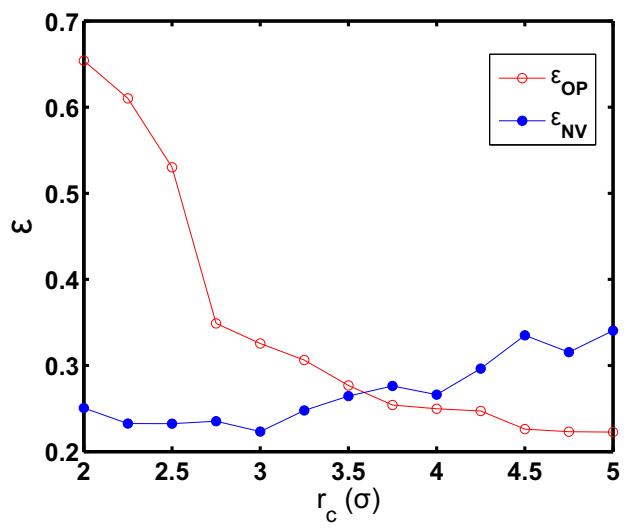

Figure 2. (a) Two perfect hexagonal grains separated by an artificial grain boundary of a known shape (black line). The sites constituting the edges of each grains are marked by red and blue stars. The interpolated sites sampling the grain boundary correspond to the magenta dots. The inset illustrates the generation of the interpolating sites in between consecutive edge sites. (b) The smoothed cloud of points $\left(r_{0}=2.5 \sigma\right)$ sampling the grain boundary. The points are coloured according to their local curvature in units of $\sigma^{-1}$ (colourbar). The arrows are the normal vectors computed using PCA. (c) Comparison between the orientation of the tangent $\beta$ found using the PCA and the analytical tangent direction. (d) The local curvature determined using the OP and NV methods together with the analytical local curvature. (e) The relative error $\epsilon$ (equation (8)) as a function of $r_{0}$ for fixed $r_{c}=2.5 \sigma$. (f) The relative error $\epsilon$ (equation (8)) as a function of $r_{c}$ for fixed $r_{0}=2.5 \sigma$. 
points shows a good consistency with the exact shape of the artificial grain boundary. The angle $\beta$ between the tangent direction and the $x$-axis is plotted in figure $2 \mathrm{c}$ and also shows excellent agreement with the value derived from the analytical interface profile, confirming the accuracy of the PCA with the chosen parameters.

In figure $2 \mathrm{~d}$ we plot the local curvature computed using the two different methods together with the analytical local curvature. Both methods show good agreement with the analytical curvature, though the OP method is more noisy and the NV method tends to underestimate large curvatures at these cut-offs. For each method, the result depends on the choice of $r_{0}$, the cut-off radius for smoothing the cloud of points sampling the grain boundary (section 3.1), and $r_{c}$, the cut-off radius for defining the neighbourhood of a point $P$ (section 2.1). To quantify this, we compute the relative error in the curvature via

$$
\epsilon=\sqrt{\frac{\left\langle\left(\kappa-\kappa_{0}\right)^{2}\right\rangle}{\left\langle\kappa_{0}^{2}\right\rangle},}
$$

where $\kappa$ is the local curvature obtained using either of the two methods and $\kappa_{0}$ is the analytical local curvature. In figure $2 \mathrm{e}$ we plot $\epsilon$ for both methods as a function of $r_{0}$ for a fixed $r_{c}=2.5 \sigma$. The OP method shows relatively large error for small $r_{0}$, which corresponds to noisy interfaces. In contrast, the NV method is not affected by the noise level of the interface and the error is relatively independent of $r_{0}$. In figure $2 \mathrm{f}$ we show the error as a function of $r_{c}$ for a fixed $r_{0}=2.5 \sigma$. For small $r_{c}$ the error in the OP method is relatively large, but rapidly decreases for increasing $r_{c}$. In contrast, the NV method shows the opposite trend as $\epsilon$ increases with $r_{c}$.

The result of the OP method is sensitive to the noise of the interface because it probes the second order variation of the interface, which becomes more accurate when the cloud of points sampling the interface is smoother, which corresponds to a large $r_{0}$ (figure 2e). Similarly, when $r_{0}$ is fixed, the method becomes more accurate when $r_{c}$ increases (figure 2f), as more points are taken into account. We note, however, that the values of $r_{0}$ and $r_{c}$ should not be larger than the typical size of geometrical features to be detected. As the NV method performs a linear analysis on the output of the PCA, which overall corresponds to two successive linear analyses, it is more robust at small cut-off distances and therefore relatively accurate for noisy interfaces (figure 2e), but underestimates large curvatures, which causes the error to increase with $r_{c}$ for fixed $r_{0}$ (figure 2f).

\section{Application to grain boundaries in $2 \mathrm{D}$ colloidal systems}

\subsection{Experimental details}

Next, we apply the method to real grain boundaries in a colloidal polycrystalline monolayer. We use melamine-formaldehyde spheres (Microparticles $\mathrm{GmbH}$ ) dispersed in a 20/80 v/v\% isopropanol/water mixture. The particles have a hard sphere diameter $\sigma$ of $2.79 \mu m$ [36]. Due to their high mass density, the particles sediment quickly to the 
(a)

(c)
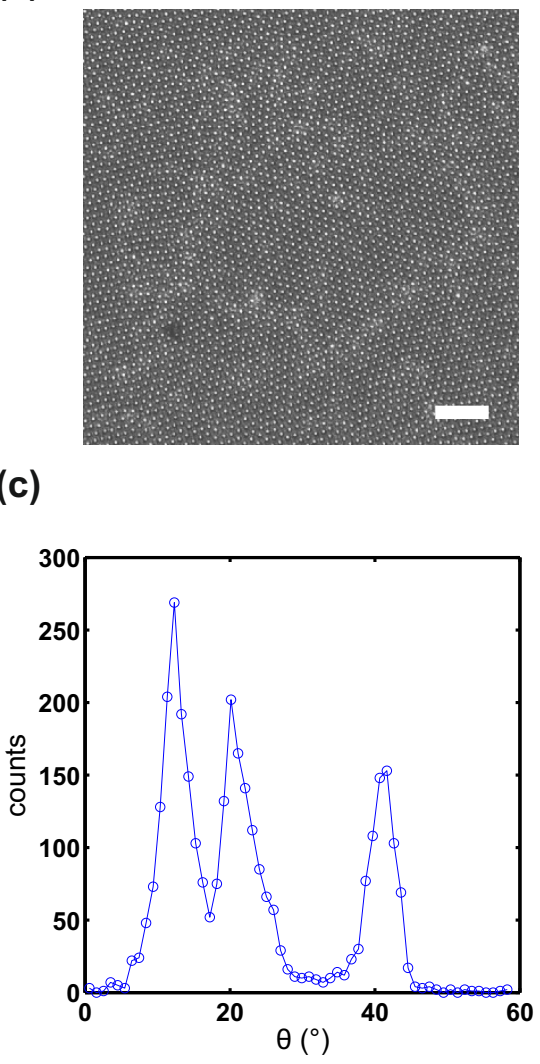

(b)
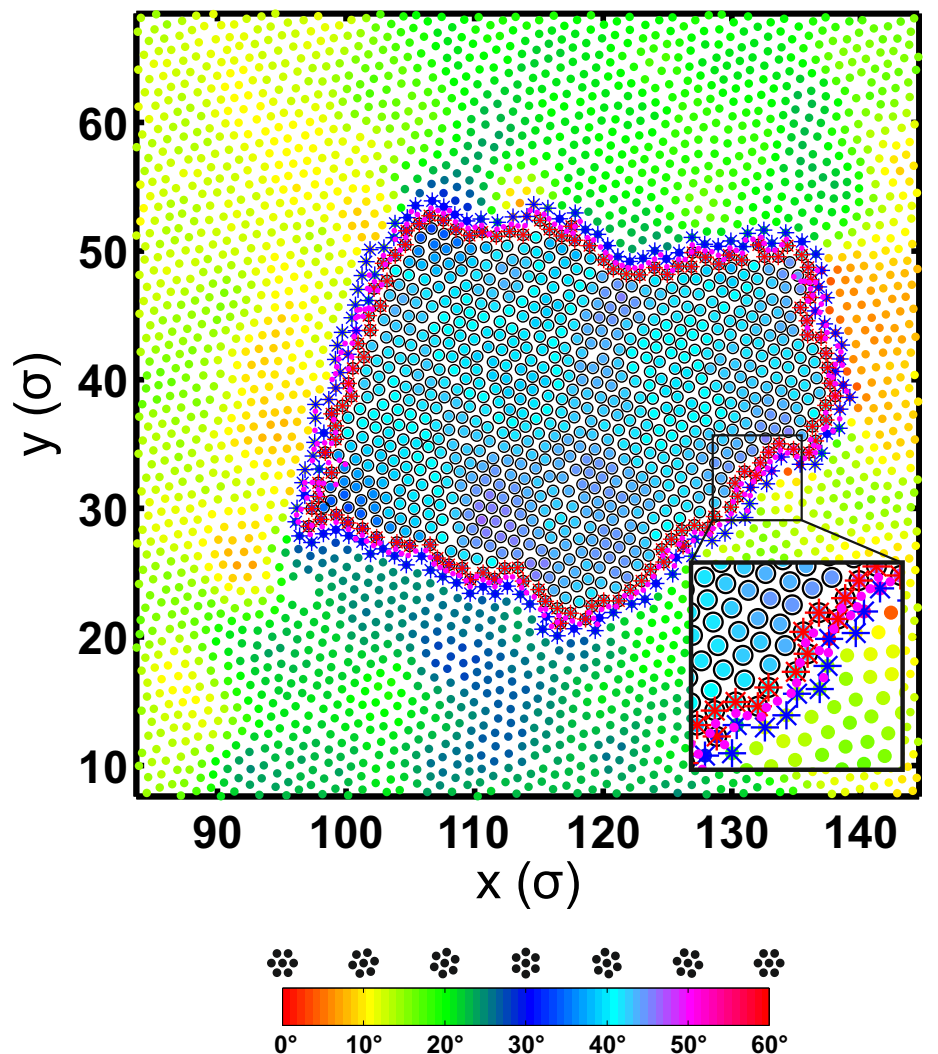

Figure 3. (a) Microscopy image of a grain in a colloidal polycrystalline monolayer. Scale bar, $20 \mu \mathrm{m}$. (b) Local orientation of the particles in panel (a) as defined by the colourbar. The central grain has an orientation $\simeq 40^{\circ}$ and its particles are circled in black. The particles constituting the edges of the central grain are marked by red stars and the ones at the edges of the surrounding grains are marked by blue stars. The interpolated sites are represented by the magenta dots (see inset). (c) Histogram of the orientations of the particles in panel (b).

bottom wall of a $200 \mu m$ thick quartz cell (Hellma Analytics) and form a polycrystalline monolayer. We image the system using video-microscopy (Olympus CKX41) and a picture of a grain is shown in figure 3a. Note that this grain is not stable and shrinks over time to eventually disappear. The coordinates of the particles are obtained using standard particle tracking routines [37].

To identify the central grain in figure $3 \mathrm{a}$ we determine the local bond orientational order parameter [38] for each particle:

$$
\psi_{6}\left(\vec{r}_{j}\right)=\frac{1}{N_{j}} \sum_{k=1}^{N_{j}} e^{i 6 \theta_{j k}},
$$

where $\theta_{j k}$ is the angle between the $x$-axis and the bond vector connecting particle $j$ at $\vec{r}_{j}$ to one of its $N_{j}$ nearest neighbours $k$, which were found using a Delaunay triangulation. Next, we replace $\psi_{6}(\vec{r}, t)$ with its average over the particles constituting the first two 
coordination shells to obtain the 'smoothed' field $\tilde{\psi}_{6}(r, t)$. This reduces the noise in the bond orientational field near grain boundaries and enables a better determination of the contour of the grains. The local orientation in the $2 \mathrm{D}$ polycrystalline system is then given by $\theta\left(\vec{r}_{j}, t\right)=\arg \left(\tilde{\psi}_{6}\left(\vec{r}_{j}, t\right)\right) / 6$ and varies from 0 to $60^{\circ}$ due to the hexagonal symmetry. A plot of the local orientation is shown in figure $3 \mathrm{~b}$ and clearly shows the central grain with an orientation $\theta_{1} \simeq 40^{\circ}$ surrounded by four other grains, two of orientation $\theta_{2} \simeq 12^{\circ}$ and two of orientation $\theta_{3} \simeq 20^{\circ}$. This is confirmed by the histogram of orientations which has three peaks around these three values (see figure 3c). We identify the central grain by selecting the particles having an orientation bigger than $30^{\circ}$ (see figure $3 \mathrm{~b}$ ).

We detect the particles corresponding to the grain boundary - and hence generate the cloud of points sampling the interface - by selecting the particles at the edge of the central grain and those at the edges of the surrounding grains (see figure $3 \mathrm{~b}$ ). As the grain is approximately circular, the cartesian coordinates are converted into the polar coordinates $(\rho, \phi)$ with respect to the centre of mass of the grain $O$ (see figure $4 \mathrm{a}$ ). As described in section 3.1, we then generate interpolating sites in between consecutive edge sites along $\phi$ to ensure that the cloud of points samples the grain boundary (see inset of figure $3 \mathrm{~b}$ ).

\subsection{Geometrical features of colloidal grain boundaries}

First, we smooth the cloud of points with a cut-off radius $r_{0}=2.5 \sigma$ (see section 3.1 ) and then we compute the normal and tangent directions of the colloidal grain boundary using PCA with a neighbourhood cut-off radius of $r_{c}=2.5 \sigma$ (see section 2.1). We position the common observation point at $O$ so that all the normals are oriented consistently. Next, the local curvature is determined using the OP and the NV methods (see section 2.2 ). In figure $4 \mathrm{~b}$ we show the smoothed cloud of points, which is coloured according to the local curvature determined using the NV method, together with the normal vectors. The local curvature obtained by both methods is shown as a function of the polar angle $\phi$ in figure 4c. Both methods give consistent local curvatures, showing that we can successfully determine the local curvature of grain boundaries from microscopy images. As was also shown for the artificial grain boundary (figure $2 \mathrm{~d}$ ), the result of the OP method is quite noisy and the output of the NV method is much smoother, yet large local curvatures may be underestimated. Interestingly, comparing figure 3b, figure $4 \mathrm{~b}$ and c reveals that the points of high local curvatures correspond to triple junctions between the central grain and two neighbouring grains. The grain boundary is relatively flat in between the triple junctions, as indicated by the numbers I, II and III in figure 4b. Also, there is a noticeable point of inflection indicated by number IV.

In figure $4 \mathrm{~d}$, we plot the tangent direction of the grain boundary $\beta$ as a function of the polar angle $\phi$. Also shown are the horizontal lines corresponding to the 6 -fold symmetric lattice directions of the inner and outer grains, $\omega_{i, k}=\theta_{i}+k \pi / 3$ where $\theta_{i}$ is the orientation of the grain corresponding to the peaks observed in figure $3 \mathrm{c}$ and $k$ is 
(a)

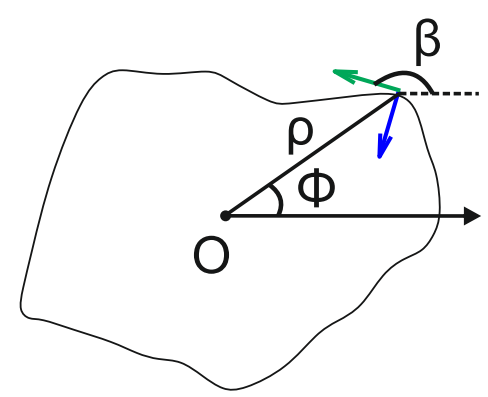

(c)

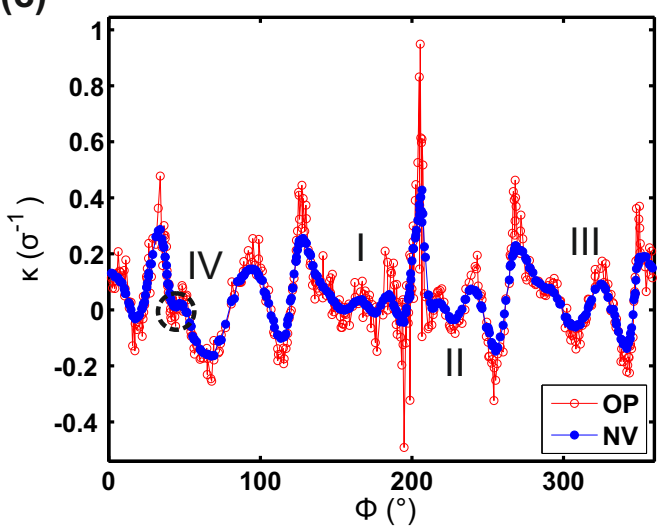

(b)

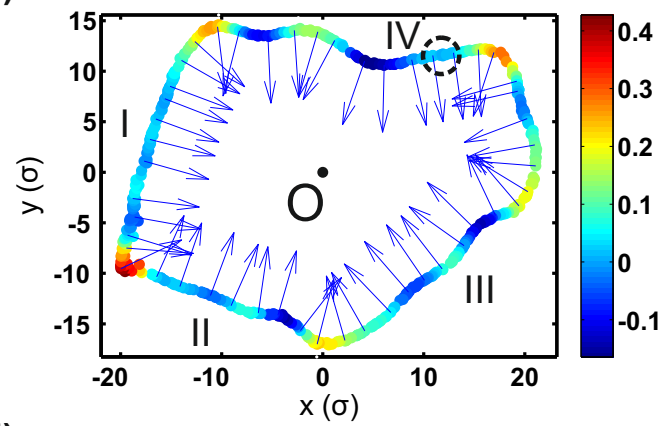

(d)

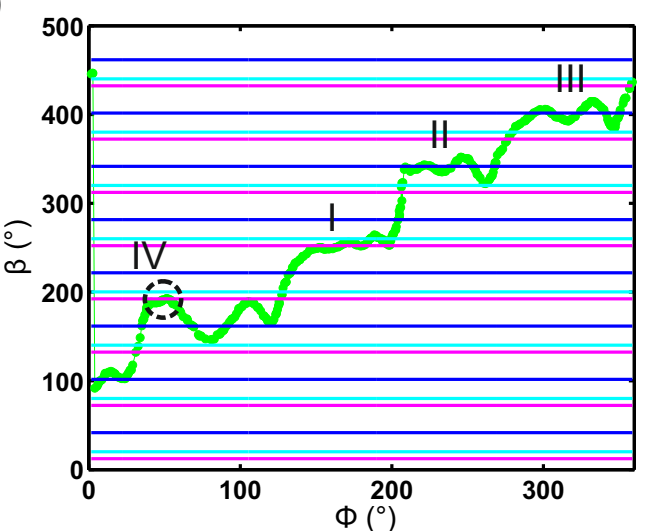

Figure 4. (a) Polar coordinates $(\rho, \phi)$ used to study the geometry of the grain. The origin $O$ corresponds to the centre of the grain. The normal and the tangent directions to the boundary correspond to the blue and green arrows. The angle in between the $x$ direction and the tangent is denoted $\beta$. (b) The smoothed cloud of points coloured according to the local curvature determined using the NV method in units of $\sigma^{-1}$ (colourbar) with several normal vectors (blue arrows). The origin $O$ is also shown. (c) Curvature of the grain boundary determined using the OP and the NV methods. (d) Orientation of the boundary as a function of the polar angle $\phi$. The horizontal lines represent the 6 -fold symmetric directions of the inner grain, $\omega_{1, k}$ (blue), and the two outer grains, $\omega_{2, k}$ and $\omega_{3, k}$ (magenta and cyan) as defined in the main text. The numbers I, II and III correspond to relatively flat edges of the grain respectively oriented along the crystal directions of an outer grain $\left(\omega_{2,4}\right)$ and of the inner grain $\left(\omega_{1,5}\right.$ and $\left.\omega_{1,6}\right)$. The number IV corresponds to an inflection point (dashed circle) where the boundary is also oriented along a crystal direction of an outer grain $\left(\omega_{2,3}\right)$.

an integer. Interestingly, the orientation of each flat part of the grain boundary - as indicated by I, II and III - is locked to the crystal direction of a grain adjacent to it and indeed corresponds to nearly zero curvatures in figure 4c. This is also observed for the inflection point - indicated by IV - in figure $4 \mathrm{~b}-\mathrm{d}$. While the equilibrium shape of a grain can be determined using the Wulff construction [1], very little is known about the shape of out of equilibrium grains. In this context, we believe the methods presented here are useful techniques to obtain new results about out of equilibrium grain boundaries studied at the single particle level. 


\section{Conclusion}

We have shown that the analysis of point sampled lines is a powerful tool to determine the local geometrical features of grain boundaries from microscopy data at the single particle level. We have presented a method to obtain local curvatures from a discrete cloud of points sampling grain boundaries. Standard Principal Component Analysis gives the normal and tangent directions while the local curvature can be determined using a local parabolic fit or the variations of the normal. We have validated the procedure using artificially generated grain boundaries and have shown that the local parabolic fit is more affected by noise, while the normal variation method tends to underestimate large curvatures. We have applied the method to grain boundaries in $2 \mathrm{D}$ colloidal systems and have found that the flat grain boundaries of a shrinking grain are aligned with one of the crystal directions of the surrounding grains. This is also true for the direction of the boundary at inflection points. As this method can be generalised to surfaces in 3D space, we believe that it can fruitfully be applied to many of the interfaces encountered in soft matter science.

\section{Acknowledgements}

The European Research Council (ERC) is acknowledged for financial support (ERC Starting Grant 279541-IMCOLMAT).

\section{Appendix A. Local curvature of a line from normal variation}

The curvature $\kappa_{P}$ of $L$ at a point $P$ of curvilinear abscissa $s_{P}$ is defined by

$$
\kappa_{P}=\left.\frac{d \theta(s)}{d s}\right|_{s=s_{P}},
$$

where $\theta(s)$ is the angle between the tangent to the curve $\vec{u}(s)$ at a point of curvilinear abscissa $s$ and the $x$-axis. In the tangent frame $\left(\vec{u}_{P}, \vec{n}_{P}\right)$ associated to $P$, we define the map $\phi$ as in equation (6) which satisfies $\phi\left(s_{P}\right)=0$. Close to $P$, one has $\theta(s)=\theta\left(s_{P}\right)+\delta \theta$ and $\phi(s)=\delta \phi$ since $\phi\left(s_{P}\right)=0$. We note that by definition of $\phi, \delta \phi=-\sin (\delta \theta) \simeq-\delta \theta$. This implies that $\frac{d \phi}{d s}=-\frac{d \theta}{d s}$ and because $d s \simeq d u$ to the lowest order, we eventually get:

$$
\begin{aligned}
\kappa_{P} & =-\left.\frac{d \phi(u)}{d u}\right|_{u=0} \\
& =-\phi^{\prime}(0) .
\end{aligned}
$$

\section{Appendix B. Extension to 3D systems}

We consider a cloud of points which samples a surface $S$ embedded in 3D space. The coordinates of the points belonging to the neighbourhood $\Omega_{P}$ of a point $P$ of the cloud 
are contained in the vectors $\mathbf{x}=\left(x_{1}, \ldots, x_{n}\right), \mathbf{y}=\left(y_{1}, \ldots, y_{n}\right)$ and $\mathbf{z}=\left(z_{1}, \ldots, z_{n}\right)$. The covariance matrix then reads $[29,30,31]$ :

$$
C_{P}=\left[\begin{array}{lll}
\operatorname{Cov}(\mathbf{x}, \mathbf{x}) & \operatorname{Cov}(\mathbf{x}, \mathbf{y}) & \operatorname{Cov}(\mathbf{x}, \mathbf{z}) \\
\operatorname{Cov}(\mathbf{y}, \mathbf{x}) & \operatorname{Cov}(\mathbf{y}, \mathbf{y}) & \operatorname{Cov}(\mathbf{y}, \mathbf{z}) \\
\operatorname{Cov}(\mathbf{z}, \mathbf{x}) & \operatorname{Cov}(\mathbf{z}, \mathbf{y}) & \operatorname{Cov}(\mathbf{z}, \mathbf{z})
\end{array}\right] .
$$

As for the $2 \mathrm{D}$ case, the normalised eigenvector associated to the lowest eigenvalue of $C_{P}$ is the normal vector $\vec{n}_{P}$ at $P$. The two other normalised eigenvectors form an orthonormal basis of the tangent plane $[29,30,31]$ and are denoted $\vec{u}_{P}$ and $\vec{v}_{P}$ in the following. A consistent orientation of the normal vectors can be obtained using the same method as in the $2 \mathrm{D}$ case. We then fix the directions of $\vec{u}_{P}$ and $\vec{v}_{P}$ such that the condition $\left(\vec{u}_{P} \times \vec{v}_{P}\right) \cdot \vec{n}_{P}>0$ is satisfied.

The OP method generalises as follows: in the local tangent frame $\left(\vec{u}_{P}, \vec{v}_{P}, \vec{n}_{P}\right)$ where the coordinates of $P$ are $(u, v, n)=(0,0,0)$, the surface $S$ is locally described by the equation $n=f(u, v)$ where $f$ is a function such that $f(0,0)=\partial_{u} f(0,0)=\partial_{v} f(0,0)=0$. Its expression to the lowest order is then given by [33]

$$
f(u, v)=\frac{1}{2}(u, v)^{T} H_{P}(u, v),
$$

where $H_{P}$ is the Hessian matrix of $f$ at $P$ defined by

$$
H_{P}=\left[\begin{array}{cc}
\partial_{u u} f & \partial_{u} \partial_{v} f \\
\partial_{u} \partial_{v} f & \partial_{v v} f
\end{array}\right] .
$$

Fitting a form $n=1 / 2\left(a u^{2}+b v^{2}+2 c u v\right)$ to the data points $(\mathbf{u}, \mathbf{v}, \mathbf{n})$ enables us to estimate the Hessian matrix of $f$ as

$$
H_{P} \simeq\left[\begin{array}{ll}
a & c \\
c & b
\end{array}\right],
$$

whose eigenvalues are estimates of $\kappa_{1}$ and $\kappa_{2}$ [33].

In the case of the NV method, the map $\phi$ which tracks the variations of the normal $\vec{n}(u, v)$ along the tangent plane at $P$, generalises as follows:

$$
\phi:\left[\begin{array}{l}
u \\
v
\end{array}\right] \mapsto\left[\begin{array}{l}
\phi_{u}(u, v) \\
\phi_{v}(u, v)
\end{array}\right]=\left[\begin{array}{l}
\vec{n}(u, v) \cdot \vec{u}_{P} \\
\vec{n}(u, v) \cdot \vec{v}_{P}
\end{array}\right] .
$$

The expression of $\phi$ to the lowest order is given by

$$
\phi(u, v)=J_{P}\left[\begin{array}{l}
u \\
v
\end{array}\right],
$$

where $J_{P}$ is the jacobian matrix of $\phi$ :

$$
J_{P}=\left[\begin{array}{cc}
\partial_{u} \phi_{u} & \partial_{v} \phi_{u} \\
\partial_{u} \phi_{v} & \partial_{v} \phi_{v}
\end{array}\right]
$$


This quantity is equivalent to the curvature tensor at $P$ [34]. Fits of $\phi_{u}=\alpha u+\beta v$ and $\phi_{v}=\gamma u+\delta v$ to the data points $\left(\mathbf{u}, \mathbf{v}, \boldsymbol{\phi}_{u}\right)$ and $\left(\mathbf{u}, \mathbf{v}, \boldsymbol{\phi}_{v}\right)$ yield an estimate of the curvature tensor as

$$
J_{P} \simeq\left[\begin{array}{ll}
\alpha & \beta \\
\gamma & \delta
\end{array}\right],
$$

whose eigenvalues are estimates of $-\kappa_{1}$ and $-\kappa_{2}$ [34].

\section{References}

[1] James M. Howe. Interfaces in materials: atomic structure, thermodynamics and kinetics of solidvapor, solid-liquid and solid-solid interfaces. Wiley-Interscience, 1997.

[2] Gunter Gottstein and Lasar S. Shvindlerman. Grain boundary migration in metals: thermodynamics, kinetics, applications. CRC press, 1999.

[3] H. Gleiter. Theory of grain boundary migration rate. Acta Metall. Mater., 17(7):853 - 862, 1969.

[4] A.E. Lobkovsky, A. Karma, M.I. Mendelev, M. Haataja, and D.J. Srolovitz. Grain shape, grain boundary mobility and the herring relation. Acta Mater., 52(2):285 - 292, 2004.

[5] Zachary T. Trautt, Moneesh Upmanyu, and Alain Karma. Interface mobility from interface random walk. Science, 314(5799):632-635, 2006.

[6] Stephen M. Foiles and J.J. Hoyt. Computation of grain boundary stiffness and mobility from boundary fluctuations. Acta Mater., 54(12):3351 - 3357, 2006.

[7] J.J. Hoyt, Z.T. Trautt, and M. Upmanyu. Fluctuations in molecular dynamics simulations. Mathematics and Computers in Simulation, 80(7):1382 - 1392, 2010. Multiscale modeling of moving interfaces in materials.

[8] Thomas O. E. Skinner, Dirk G. A. L. Aarts, and Roel P. A. Dullens. Grain-boundary fluctuations in two-dimensional colloidal crystals. Phys. Rev. Lett., 105:168301, Oct 2010.

[9] K.L. Merkle and L.J. Thompson. Atomic-scale observation of grain boundary motion. Mater. Lett., 48(34):188 - 193, 2001.

[10] Pinshane Y. Huang, Carlos S. Ruiz-Vargas, Arend M. van der Zande, William S. Whitney, Mark P. Levendorf, Joshua W. Kevek, Shivank Garg, Jonathan S. Alden, Caleb J. Hustedt, Ye Zhu, et al. Grains and grain boundaries in single-layer graphene atomic patchwork quilts. Nature, 469(7330):389-392, 2011.

[11] Simon Kurasch, Jani Kotakoski, Ossi Lehtinen, Viera Skkalov, Jurgen Smet, Carl E. Krill, Arkady V. Krasheninnikov, and Ute Kaiser. Atom-by-atom observation of grain boundary migration in graphene. Nano Lett., 12(6):3168-3173, 2012. PMID: 22554303.

[12] Thomas O. E. Skinner, Dirk G. A. L. Aarts, and Roel P. A. Dullens. Supercooled dynamics of grain boundary particles in two-dimensional colloidal crystals. J. Chem. Phys., 135(12):124711, 2011.

[13] A. R. Bausch, M. J. Bowick, A. Cacciuto, A. D. Dinsmore, M. F. Hsu, D. R. Nelson, M. G. Nikolaides, A. Travesset, and D. A. Weitz. Grain boundary scars and spherical crystallography. Science, 299(5613):1716-1718, 2003.

[14] A. M. Alsayed, M. F. Islam, J. Zhang, P. J. Collings, and A. G. Yodh. Premelting at defects within bulk colloidal crystals. Science, 309(5738):1207-1210, 2005.

[15] Peter Lipowsky, Mark J. Bowick, Jan H. Meinke, David R. Nelson, and Andreas R. Bausch. Direct visualization of dislocation dynamics in grain-boundary scars. Nature Mater., 4(5):407-411, 2005 .

[16] Lee J. Moore, Richard D. Dear, Michael D. Summers, Roel P. A. Dullens, and Grant A. D. Ritchie. Direct observation of grain rotation-induced grain coalescence in two-dimensional colloidal crystals. Nano Letters, 10(10):4266-4272, 2010. PMID: 20853830.

[17] Volkert W. A. de Villeneuve, Leonie Derendorp, Danny Verboekend, Esther C. M. Vermolen, 
Willem K. Kegel, Henk N. W. Lekkerkerker, and Roel P. A. Dullens. Grain boundary pinning in doped hard sphere crystals. Soft Matter, 5(12):2448-2452, 2009.

[18] K Hima Nagamanasa, Shreyas Gokhale, Rajesh Ganapathy, and A.K. Sood. Confined glassy dynamics at grain boundaries in colloidal crystals. Proc. Natl Acad. Sci. USA, 108(28):1132311326, 2011.

[19] Shreyas Gokhale, K. Hima Nagamanasa, Rajesh Ganapathy, and A.K. Sood. Grain growth and grain boundary dynamics in colloidal polycrystals. Soft Matter, 9:6634-6644, 2013.

[20] Hao Zhang, David J. Srolovitz, Jack F. Douglas, and James A. Warren. Grain boundaries exhibit the dynamics of glass-forming liquids. Proc. Natl Acad. Sci. USA, 106(19):7735-7740, 2009.

[21] Dirk G. A. L. Aarts, Matthias Schmidt, and Henk N. W. Lekkerkerker. Direct visual observation of thermal capillary waves. Science, 304(5672):847-850, 2004.

[22] Meghan K. Driscoll, Colin McCann, Rael Kopace, Tess Homan, John T. Fourkas, Carole Parent, and Wolfgang Losert. Cell shape dynamics: from waves to migration. PLoS computational biology, 8(3):e1002392, 2012.

[23] M. A. Martorano, M. A. Fortes, and A. F. Padilha. A numerical method for curvature driven boundary migration. Modelling and Simulation in Materials Science and Engineering, 14(1):83, 2006 .

[24] James A. Sethian. A fast marching level set method for monotonically advancing fronts. Proc. Natl Acad. Sci. USA, 93(4):1591-1595, 1996.

[25] Jessica Hernández-Guzmán and Eric R. Weeks. The equilibrium intrinsic crystal-liquid interface of colloids. Proc. Natl Acad. Sci. USA, 106(36):15198-15202, 2009.

[26] C Patrick Royall, Dirk G. A. L. Aarts, and Hajime Tanaka. Bridging length scales in colloidal liquids and interfaces from near-critical divergence to single particles. Nature Phys., 3(9):636640, 2007.

[27] Van D. Nguyen, Zhibing Hu, and Peter Schall. Single crystal growth and anisotropic crystal-fluid interfacial free energy in soft colloidal systems. Phys. Rev. E, 84:011607, Jul 2011.

[28] Hiroshi Noguchi and Gerhard Gompper. Meshless membrane model based on the moving leastsquares method. Phys. Rev. E, 73:021903, Feb 2006.

[29] J. Berkmann and T. Caelli. Computation of surface geometry and segmentation using covariance techniques. Pattern Analysis and Machine Intelligence, IEEE Transactions on, 16(11):11141116, Nov 1994.

[30] J. Daniels, L.K. Ha, T. Ochotta, and C.T. Silva. Robust smooth feature extraction from point clouds. In Shape Modeling and Applications, 200\%. SMI '0\%. IEEE International Conference on, pages 123-136, June 2007.

[31] Hugues Hoppe, Tony DeRose, Tom Duchamp, John McDonald, and Werner Stuetzle. Surface reconstruction from unorganized points. SIGGRAPH Comput. Graph., 26(2):71-78, July 1992.

[32] R.B. Rusu, N. Blodow, Z.C. Marton, and M. Beetz. Aligning point cloud views using persistent feature histograms. In Intelligent Robots and Systems, 2008. IROS 2008. IEEE/RSJ International Conference on, pages 3384-3391, Sept 2008.

[33] Sylvain Petitjean. A survey of methods for recovering quadrics in triangle meshes. ACM Comput. Surv., 34(2):211-262, June 2002.

[34] E. Kalogerakis, P. Simari, D. Nowrouzezahrai, and K. Singh. Robust statistical estimation of curvature on discretized surfaces [article]. In Proceedings of the fifth Eurographics Symposium on Geometry Processing Eurographics Association, page 22, 2007.

[35] Mark Pauly, Markus Gross, and Leif P. Kobbelt. Efficient simplification of point-sampled surfaces. In Proceedings of the Conference on Visualization '02, VIS '02, pages 163-170, Washington, DC, USA, 2002. IEEE Computer Society.

[36] Alice L. Thorneywork, Roland Roth, Dirk G. A. L. Aarts, and Roel P. A. Dullens. Radial distribution functions in a two-dimensional binary colloidal hard sphere system. J. Chem. Phys., 140(16):161106, 2014.

[37] John C. Crocker and David G. Grier. Methods of digital video microscopy for colloidal studies. 
J. Colloid Interf. Sci., 179(1):298 - 310, 1996.

[38] David R. Nelson. Defects and geometry in condensed matter physics. Cambridge University Press, 2002. 